\title{
A direct cerebello-telencephalic projection in an electrosensory mormyrid fish
}

\author{
Mario F. Wullimann ${ }^{1}$ and Donal J. Rooney ${ }^{2 *}$ \\ ${ }^{I}$ Georg-August Universität, Zentrum Anatomie, Göttingen (F.R.G.) and ${ }^{2}$ Département de Neurophysiologie sensorielle, Laboratoire de \\ Physiologie nerveuse, C.N.R.S., Gif-sur-Yvette (France)
}

(Accepted 6 March 1990)

Key words: Cerebellum; Electroreception; Fish; Mormyrid; Telencephalon; Teleost

\begin{abstract}
After injections of the posterior part of the lateral zone of the area dorsalis telencephali (Dlp) with either horseradish peroxidase or the newly available carbocyanine dye DiI, efferent cells were labeled in the valvula cerebelli of the mormyrid fish, Gnathonemus petersii. This may be a unique connection for this group of electrosensory teleosts, since no other vertebrate has ever been reported before to have a direct cerebello-telencephalic projection.
\end{abstract}

The complex brain of mormyrids has attracted the attention of neuroscientists for almost 150 years $^{3}$. Recently, it has been estimated that the brain weight/body weight ratio in mormyrid fish is similar to humans $(1: 50)^{19}$. The extraordinary development of the valvula, a subdivision of the cerebellum unique to ray-finned fishes, accounts for much of the large brain volume. The valvula cerebelli is hypertrophied in the African mormyrids to such a degree that it envelopes most of the rest of the brain ${ }^{14}$. Furthermore, the telencephalon of mormyrid fish ranges among the largest and most highly differentiated ones of all teleosts ${ }^{13,20}$. The discovery that mormyrids are electrosensory ${ }^{9}$ resulted in speculation that the large valvula might process electrosensory information. Indeed, subsequent neurophysiological ${ }^{18}$ and neuroanatomical ${ }^{6,10}$ studies on mormyrids confirmed that parts of the valvula are related to the electrosensory system. However, there was no indication of a valvular projection to the telencephalon in these studies ${ }^{6,10}$.

Data on telencephalic connections in mormyrids only recently became available when the afferent and efferent connections of many of the telencephalic subdivisions in

Fig. 1. Cross-sections through the valvula cerebelli showing (a) the location of a retrogradely filled cell within the inner valvular leaf after an injection of HRP into the telencephalon. Note the granular label in the cell body and axon. b: a lower power microphotograph of the retrogradely labelled cell (arrowheads) shown in a. Note size of labelled cell and its position within the intermediate layer. Lateral is to the left. $\mathrm{g}$, granular layer; $\mathrm{m}$, molecular layer; $\mathrm{i}$, intermediate layer (containing centrally the Purkinje cell layer). Bars $=0.1 \mathrm{~mm}$.
Gnathonemus were investigated with the HRP and/or the cobalt techniques ${ }^{17,21}$ (Rooney, in preparation; Wullimann and Northcutt, J. Comp. Neurol., submitted).
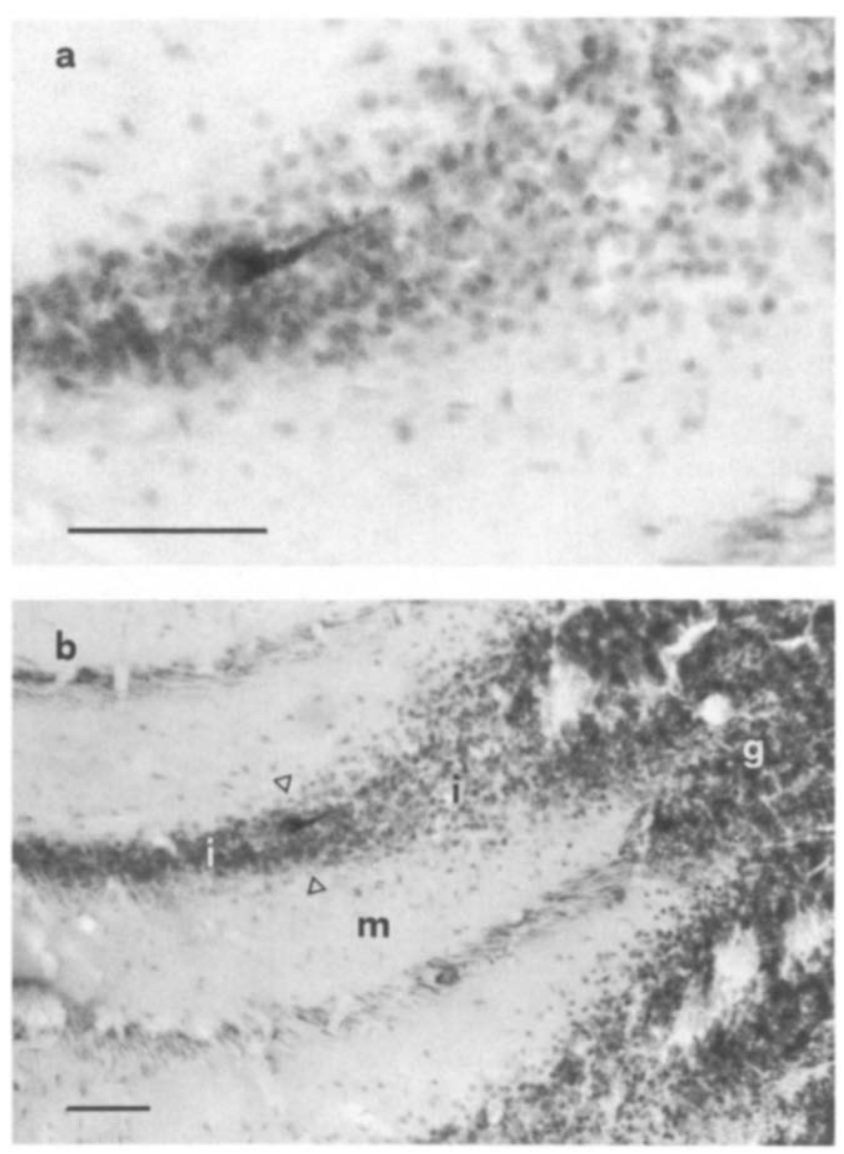

* Present address: Institut für Zoologie, Technische Hochschule, Schnittspahnstr. 3, D-6100 Darmstadt, F.R.G.

Correspondence: M.F. Wullimann, Georg-August Universität, Zentrum Anatomie, Kreuzbergring 36, D-3400 Göttingen, F.R.G. 

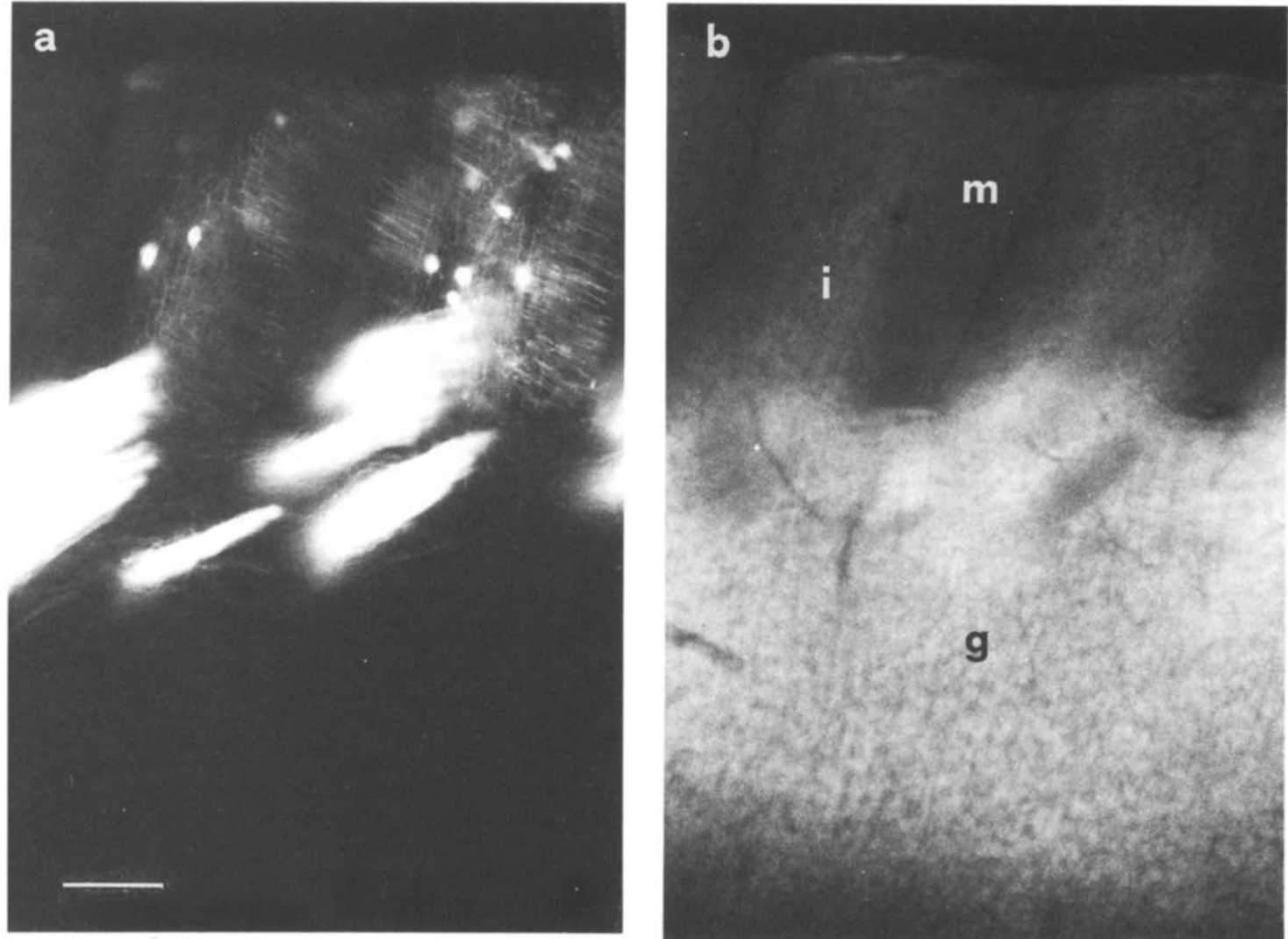

Fig. 2. Cross-sections through the valvula cerebelli showing (a) the labelled fiber bundles and efferent valvular cells (basal cells and vertical cells, see text) in the ipsilateral valvular ridges of Gnathonemus after a DiI injection into the telencephalon. b: the same section emphasizing the valvular histology with the fluorescent Nissl-like counterstain $m$-phenylenediamine. Abbreviations as in Fig. 1. Lateral is to the top. Bar $=0.1 \mathrm{~mm}$.
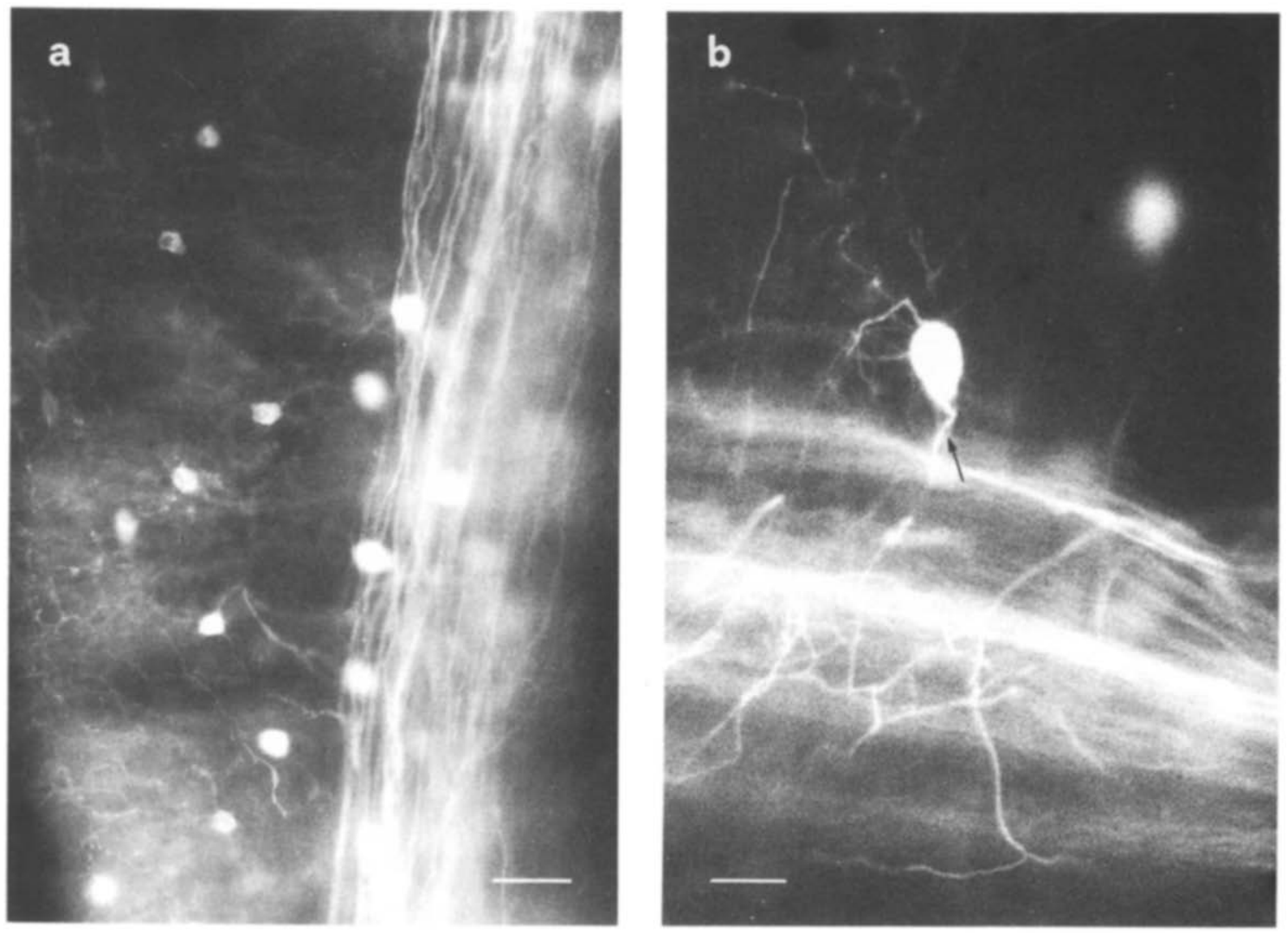

Fig. 3. Cross-section through a valvular ridge of Gnathonemus showing (a) many labelled cells (lateral is to the left), and (b) one enlarged central cell (lateral is to the top) after a DiI injection into the telencephalon. Arrow points to the axon. Bar $=0.05 \mathrm{~mm}$ in $\mathrm{a} ;=0.025 \mathrm{~mm}$ in $b$. 

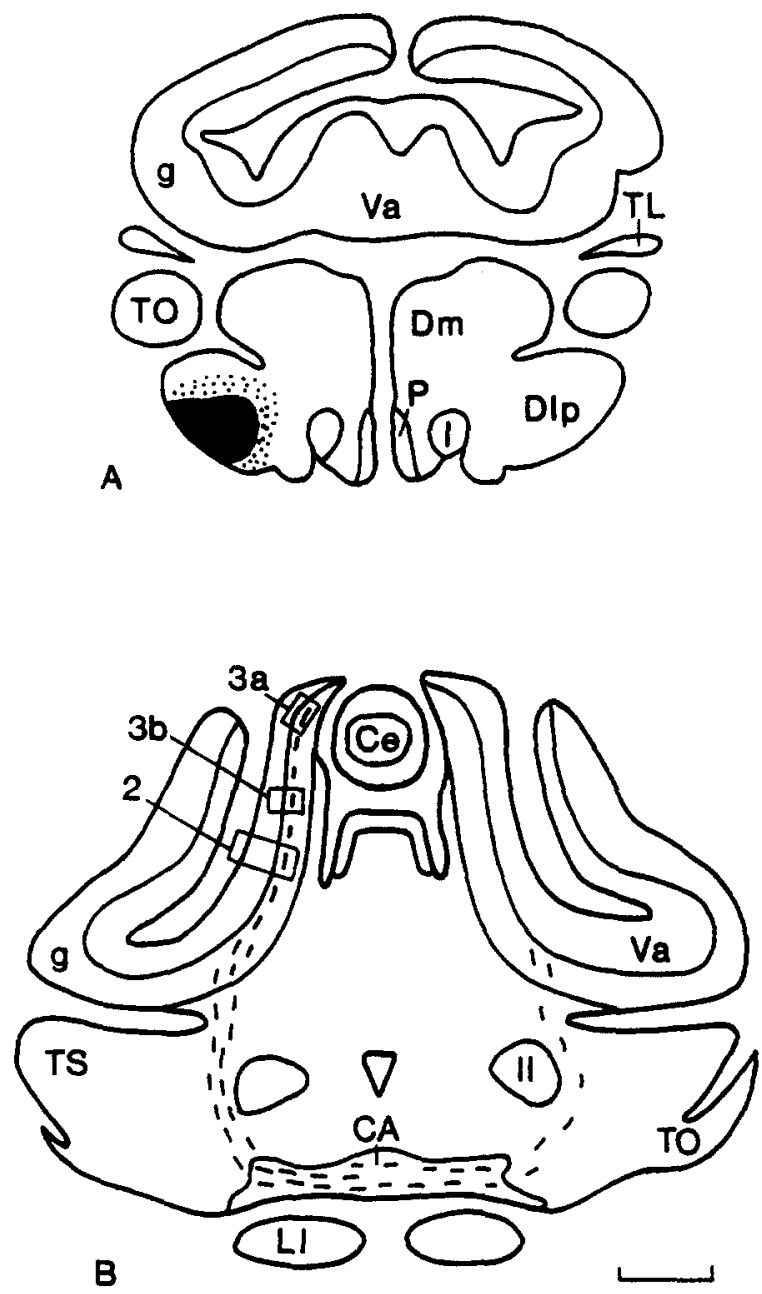

Fig. 4. Drawings of cross-sections through the brain of Gnathonemus illustrating (A) the DiI injection site (black spot) in the telencephalon, and (B) the course of labelled fibers (dashes) in the valvula. Squares indicate location of photomicrographs of Figs. 2, 3a and 3b. CA, commissura ansulata; Ce, corpus cerebelli; Dlp, posterior part of lateral zone of area dorsalis telencephali; Dm, medial zone of area dorsalis telencephali; $\mathrm{g}$, granular layer; $\mathbf{l}$, lateral forebrain bundle; LI, inferior lobe of the hypothalamus; II, lateral lemniscus; P, preoptic area; TL, torus longitudinalis; TO, tectum opticum; TS, torus semicircularis; Va, valvula cerebelli. Bar $=1$ $\mathrm{mm}$.

HRP injections, which included the posterior part of the lateral zone of area dorsalis telencephali (Dlp; see Fig. $4 \mathrm{~A}$ ), provided a first indication of a connection with the valvula, as labelled fibers could be observed entering the valvular peduncle (Rooney, in preparation). These labelled fibers could not be observed after injections of HRP into more rostral telencephalic areas.

To consolidate these preliminary findings we anesthetized specimens of Gnathonemus with MS 222 (Sigma) and selectively injected Dlp with HRP (Boehringer, grade 1 or Sigma, grade VI). After 2-7 days the animals were perfused and fixed in $4 \%$ glutaraldehyde. The HRP was visualized according to the protocol of Ebbesson et al. ${ }^{1}$ or of Hanker et al. ${ }^{8}$. Additional anesthetized speci- mens were perfused and fixed in $4 \%$ paraformaldehyde and Dlp was injected with crystals of the carbocyanine dye DiI (Molecular Probes, OR). The brains were then incubated in the fixative for $47-100$ days before being vibratome-sectioned at $50 \mu \mathrm{m}$.

Following HRP-injections into Dlp, labelled fibers were observed in the ipsilateral cerebellar peduncle and a few retrogradely filled cells could be seen in many ridges of the inner leaf of the ipsilateral valvula (Fig. 1). The Dil injections into Dlp not only confirmed these results, but revealed the full extent of the valvulotelencephalic connection. In the Dil cases massive fiber bundles were labelled in the ipsilateral valvular peduncle (Figs. 2, 4B) and many large neurons were retrogradely labelled all along the course of these fibers (Figs. 2A, 3) within the inner leaf of the ipsilateral valvula. Based on their topological position within the valvular ridges and their dendritic morphology, these labelled cells probably include representatives of all 3 types of valvular efferent neurons (basal, vertical and central) described by Nieuwenhuys and Nicholson ${ }^{15}$. At the bottom of the valvular ridges, large basal cells can be observed, whereas more apically smaller vertical cells are labelled (Fig. 2). Central cells with less regularly arranged dendrites appear also to be labelled (Fig. 3). Labelled fibers were observed to cross in the commissura ansulata and to enter the contralateral valvular peduncle (Fig. 4B). However, no retrogradely labelled cells were observed in the contralateral valvula, and therefore a decision on whether these contralateral fibers are afferent or efferent could not be made. While the principal telencephalic target of the ascending cerebellar projection appears to be Dlp, further studies are needed to elucidate how localized the valvular projection is within the telencephalon.

In summary, the present data suggest that mormyrids may be unique among vertebrates in that they possess a direct cerebello-telencephalic projection; as such, this connection has never been reported before ${ }^{16}$. It seems unlikely that the absence of a cerebello-telencephalic projection in previous studies on cerebellar ${ }^{4-6,10,11,19,22,23}$ and telencephalic ${ }^{2,12}$ connections in teleosts is due to the fact that different methodologies were used, as we could label the connection with both HRP and DiI. However, the application of the newly available DiI methodology? allows one to trace neuronal connections in vitro for much longer distances than is possible with the in vivo techniques (HRP, cobalt, autoradiography). Therefore, studies in non-electrosensory teleosts with $\mathrm{DiI}$ should reveal if the cerebello-telencephalic connection is primitive for teleosts or if it is a neuroanatomical feature newly acquired during the evolution of electroreception in mormyrids. The latter possibility is suggested by both neuroanatomical ${ }^{6}$ and electrophysiological ${ }^{18}$ findings in 
mormyrids which demonstrate that the cerebellar regions related to electroreception are in the medial and ventrolateral areas of the inner leaf of the valvula (the mormyromast-ampullary region of Finger et al. ${ }^{6}$ ). Many of the retrogradely filled cells were found within this region.

Furthermore, it was recently argued that extensive portions of the electrosensory-related neuronal circuitry between the telencephalon, diencephalon and cerebellum in mormyrids are derived features for this group as they do not exist in non-electrosensory teleosts ${ }^{21,23}$. This was interpreted as the neuroanatomical consequence of the evolution of electroreception as a new sensory modality in this teleost group. The unexpected cerebello-telence-

1 Ebbesson, S.O.E., Hansel, M. and Scheich, H., An 'on the slide' modification of the DeOlmos-Heimer HRP method, Neurosci. Lett., 22 (1981) 1-14.

2 Echteler, S.M. and Saidel, W.M., Forebrain connections in the goldfish support telencephalic homologies with land vertebrates, Science, 212 (1981) 683-685.

3 Erdl, M.P., Über das Gehirn der Fischgattung Mormyrus, Gel. Anz. Königl.-Bayer. Akad. Wissensch., 23 (1846) 403-407.

4 Finger, T.E., Cerebellar afferents in teleost catfish (Ictaluridae), J. Comp. Neurol., 181 (1978) 173-182.

5 Finger, T.E., Efferent neurons of the teleost cerebellum, Brain Research, 153 (1978) 608-614.

6 Finger, T.E., Bell, C.C. and Russell, C.J., Electrosensory pathways to the valvula cerebelli in mormyrid fish, Exp. Brain Res., 42 (1981) 23-33.

7 Godement, P., Vanselow, J., Thanos, S. and Bonhoeffer, F., A study in developing visual systems with a new method of staining neurones and their processes in fixed tissue, Development, 101 (1987) 697-713.

8 Hanker, J.S., Yates, P.E., Metz, C.B. and Rustioni, A., A new specific, sensitive and non-carcinogenic reagent for the demonstration of horseradish-peroxidase, Histochem. J., 9 (1977) 789-792.

9 Lissmann, H.W., On the function and evolution of electric organs in fish, J. Exp. Biol., 35 (1958) 156-191.

10 Meek, J., Nieuwenhuys, R. and Elsevier, D., Afferent and efferent connections of cerebellar lobe $\mathrm{C} 1$ of the mormyrid fish Gnathonemus petersi: an HRP study, J. Comp. Neurol., 245 (1986) 319-341.

11 Murakami, T. and Morita, Y., Morphology and distribution of the projection neurons in the cerebellum in a teleost, Sebastiscus marmoratus, J. Comp. Neurol., 256 (1987) 607-623.

12 Murakami, T., Morita, Y. and Ito, H., Extrinsic and intrinsic fiber connections of the telencephalon in a teleost, Sebastiscus marmoratus, J. Comp. Neurol., 216 (1983) 115-131. phalic projection reported here in Gnathonemus may have the same evolutionary explanation.

The functional significance of the cerebello-telencephalic projection needs to be determined. Does the valvula simply provide higher order sensory information to the telencephalon or does it exert some control over the telencephalon? Answering these questions remains an exciting field to be investigated.

We thank S. Kötting and K. Sebralla for technical assistance, Dr. T. Szabo for critically reading the manuscript and the Swiss National Science Foundation (M.F.W.) and the Royal Society, European exchange fellowship and Foundation pour la Recherche Médicale Française (D.J.R.) for financial support.

13 Nieuwenhuys, R., The comparative anatomy of the actinopterygian forebrain, J. Hirnforsch., 6 (1963) 171-192.

14 Nieuwenhuys, R. and Nicholson, C., A survey of the general morphology, the fiber connections, and the possible functional significance of the gigantocerebellum of mormyrid fishes. In R. Llinas (Ed.), Neurobiology of Cerebellar Evolution and Development, Am. Med. Assoc., Chicago, 1969, pp. 107-134.

15 Nieuwenhuys, R. and Nicholson, C., Aspects of the histology of the cerebellum of mormyrid fishes. In R. Llinas (Ed.), Neurobiology of Cerebellar Evolution and Development, Am. Med. Assoc., Chicago, 1969, pp. 135-169.

16 Northcutt, R.G., Evolution of the telencephalon in nonmammals, Annu. Rev. Neurosci., 4 (1981) 301-350.

17 Rooney, D.J., New, J.G., Szabo, T. and Ravaille-Veron, M., Central connections of the olfactory bulb in the weakly electric fish, Gnathonemus petersii, Cell Tiss. Res., 257 (1989) 423-436.

18 Russell, C.J. and Bell, C.C., Neuronal responses to electrosensory input in mormyrid valvula cerebelli, $J$. Neurophysiol., 41 (1978) 1495-1510.

19 Szabo, T., Cerebellar pathways in the brain of mormyrid teleost fish, Acta Morphol. Hung., 31 (1983) 219-234.

20 Weston, J.K., Notes on the telencephalon of Mormyrus and Gnathonemus, Kon. Akad. v. Wetensch. Amsterdam Proc. Sect. Sci., 40 (1937) 894-904.

21 Wullimann, M.F. and Northcutt, R.G., Telencephalic and retinal projections allow reinterpretation of the diencephalon in mormyrids, Soc. Neurosci. Abstr., 13 (1987) 130.

22 Wullimann, M.F. and Northcutt, R.G., Connections of the corpus cerebelli in the green sunfish and the common goldfish: a comparison of perciform and cypriniform teleosts, Brain Behav. Evol., 32 (1988) 293-316.

23 Wullimann, M.F. and Northcutt, R.G., Afferent connections of the valvula cerebelli in two teleosts, the common goldfish and the green sunfish, J. Comp. Neurol., 289 (1989) 554-567. 